\title{
- Towards more robust estimates of the transmissibility of Chlamydia trachomatis
}

3 Christian L. Althaus, PHD, Janneke C.M. Heijne, MS, and Nicola Low, MD

Institute of Social and Preventive Medicine (ISPM)

University of Bern, 3012 Bern, Switzerland

5 Post-print: Original version published in Sex Transm Dis. 2012 May;39(5):402-4.

6

7 DOI: http://dx.doi.org/10.1097/OLQ.0b013e318248a550

9 Potential conflicts of interest: none reported.

10

11 Correspondence: Dr. Christian L. Althaus, Institute of Social and Preventive Medicine 12 (ISPM), University of Bern, Finkenhubelweg 11, 3012 Bern, Switzerland 13 (christian.althaus@alumni.ethz.ch).

14

15 Funding: This work was supported by the NIHR Health Technology Assessment programme 16 (project number 07/42/02). The project will be published in full in the Health Technology As17 sessment journal series. Visit the HTA programme website for more details 18 (www.hta.ac.uk/1722). The views and opinions expressed therein are those of the authors and 19 do not necessarily reflect those of the Department of Health. JCMH received funding from the

20 Swiss National Science Foundation (project number 320030-118424 and 320030-135654). 
Summary

This study provides improved estimates of the transmissibility of Chlamydia trachomatis in heterosexual partnerships, using a mathematical model that considers the dynamics of chlamydia transmission and sexual partnership formation. 
Knowledge about the transmissibility of Chlamydia trachomatis (chlamydia) is important for health professionals to be able to give accurate information to their patients and for those investigating and implementing preventive interventions. ${ }^{1}$ Estimates of transmission probabilities are needed in research studies to parameterize mathematical and computational models, which are used to study the transmission dynamics of chlamydia and the impact of public health interventions such as screening programs. ${ }^{2}$ Chlamydia is the most commonly diagnosed bacterial sexually transmitted infection in many developed countries. Prevalence is high with more than 2 million infected persons per year in the USA alone. ${ }^{3}$ It is, however, difficult to obtain reliable estimates of chlamydia transmissibility from observational epidemiological studies.

Empirical estimates of the transmissibility of chlamydia have usually been based on data about the proportions of concordant and discordant pairs that are infected or uninfected with chlamydia. ${ }^{4}$ Katz proposed an original approach for analyzing such couple data. ${ }^{5}$ The expected numbers of concordant and discordant couples before transmission takes place can be calculated if it is assumed that all couples in the population with at least one infected individual have the same probability of observation and that sexual partnership formation is independent of infection status. After sexual partnerships have formed, transmission can happen in discordant partnerships resulting in a higher proportion of couples where both partners are positive. Using data from heterosexual couples attending a sexually transmitted diseases (STD) clinic in Indianapolis, Katz estimated the probabilities that transmission has occurred within a couple at 0.395 from men to women and 0.323 from women to men. There are two major problems with this approach, however. First, the infection status of the couples is observed during the partnership and not at the end so the estimated transmission probabilities do not represent the per partnership transmission probability; this frequently used concept implies the probability of transmission by the end of the partnership. Second, it does not take into account the natural history of chlamydia infection where spontaneous clearance and re-infection within sexual partnerships can occur. ${ }^{6}$ These additional complexities need to be considered because different assumptions about the infectious duration and re-infection in sexual partnerships can affect the prevalence of chlamydia. ${ }^{7,8}$

In this study, we apply a mathematical model to data from a cross-sectional partnership study that has frequently been used as the source of estimates for chlamydia transmissibility. ${ }^{9}$ Mathematical models are a tool for explicitly describing the dynamics of sexual partnership 
formation and transmission of chlamydia infection. The pair model framework has proven useful for describing the transmission of chlamydia and other STIs. ${ }^{8,10-12}$ The model considers the formation of sexual partnerships or pairs $(P)$ and their dissolution into singles $(X)$. Quinn et al. studied heterosexual couples attending two STD clinics in Baltimore. ${ }^{9}$ There were comparable numbers of discordant couples where either the woman or man was infected, so we made the simplifying assumption that the prevalence and the natural history of chlamydia infection were the same in women and men. Assuming a SIS (susceptible-infected-susceptible) structure, the pair formation and chlamydia transmission can be described by the following set of ordinary differential equations:

$$
\begin{aligned}
\frac{d X_{S}}{d t} & =-\rho X_{S}+\gamma X_{I}+2 \sigma P_{S S}+\sigma P_{S I}, \\
\frac{d X_{I}}{d t} & =-\rho X_{I}-\gamma X_{I}+2 \sigma P_{I I}+\sigma P_{S I}, \\
\frac{d P_{S S}}{d t} & =\frac{1}{2} \rho \frac{X_{S}^{2}}{X}+\gamma P_{S I}-\sigma P_{S S}, \\
\frac{d P_{S I}}{d t} & =\rho \frac{X_{S} X_{I}}{X}-f \beta P_{S I}+2 \gamma P_{I I}-\gamma P_{S I}-\sigma P_{S I}, \\
\frac{d P_{I I}}{d t} & =\frac{1}{2} \rho \frac{X_{I}^{2}}{X}+f \beta P_{S I}-2 \gamma P_{I I}-\sigma P_{I I} .
\end{aligned}
$$

The infection status is represented by the indices $S$ and $I$ for being susceptible or infected, respectively. Singles $X$ seek partners at rate $\rho$ resulting in sexual partnerships with a concordant $\left(P_{S S}\right.$ and $\left.P_{I I}\right)$ or discordant $\left(P_{S I}\right)$ chlamydia infection status. The duration of sexual partnerships and infection are exponentially distributed with a mean duration of $1 / \sigma$ and $1 / \gamma$, respectively. Within discordant partnerships, couples engage in heterosexual intercourse at rate $f$ and transmission happens with probability $\beta$ per sex act. The per partnership transmission probability $p$ is defined as the probability that an infected individual transmits chlamydia to his or her susceptible partner before the infection is spontaneously cleared or the partnership breaks up $(p=f \beta /(\sigma+\gamma+f \beta))$.

We assumed that chlamydia infection in the population had approached steady-state, i.e., all derivates can be set to zero. We then used maximum likelihood estimation (MLE) ${ }^{13}$ to fit the model to the data from the study by Quinn et al. ${ }^{9}$ The study contains information about chlamydia infection status and sexual activity in 494 heterosexual couples. The study reported 53 concordant chlamydia positive, 48 discordant and 393 concordant negative couples. The median number of sex partners in the last 6 months was reported to be 1 in females and 2 
in males. In the model, we account for a skewed distribution and vary the mean number of partners during the previous 6 months for individuals in a partnership at steady-state between 1.5 and 3. The median number of episodes of sexual intercourse in the preceding 30 days was reported to be 6 , so we assumed one sex act every 5 days. Due to uncertainties in the duration of sexual partnerships and infections, we generated 1000 parameter combinations by sampling the mean partnership duration from a uniform distribution between 1 week and the maximal duration that is consistent with the assumed number of partners in the last 6 months, and the infectious duration from a uniform distribution between 6 and 12 months. ${ }^{7}$ Note that infectious durations of more than 12 months resulted in poor descriptions of the data.

The pairs in the model represent the couples from the study by Quinn et al. ${ }^{9}$. The singles can be interpreted as the population from which they are drawn. Assuming that the test data are binomially distributed, we fitted the model to the overall positivity of all individuals in couples (154 of 988) and to the positivity of partners of index patients that are either chlamydia positive (53 of 77 ) or negative (24 of 417). Using the function mle from the $\mathrm{R}$ software environment for statistical computing, ${ }^{14}$ we estimated the per sex act transmission probability $\beta$ from which we also derived the per partnership transmission probability $p$. Estimates that resulted in per sex act transmission probabilities of more than $100 \%$ or low goodness of fit based on the Akaike information criterion (AIC) ${ }^{15}$ were excluded.

We obtained model estimates of the per partnership transmission probability for different values of the number of partners during the last 6 months (Fig. 1A). Higher number of partners resulted in lower estimates of the per partnership transmission probability. However, partner numbers of 3 or more during the last 6 months resulted in poor fits to the data. We therefore consider 2 partners during the last 6 months as our baseline scenario for which the median of the estimated per partnership transmission probability is $55.5 \%$ (interquartile range (IQR): $49.2 \%-62.5 \%)$. The estimates of the per sex act transmission probability seemed to be less affected by the assumed number of partners in the last 6 months (Fig. 1B). Most values were around $10 \%$ with the median of the baseline scenario at 9.5\% (IQR: $6.0 \%-16.7 \%$ ).

Our estimated range of chlamydia transmission probabilities in heterosexual partnerships is higher than the baseline values reported by Katz. ${ }^{5}$ This is expected because we report the probability of transmission taking place by the end of a partnership. The additional assumption that chlamydia can be cleared spontaneously also results in higher estimates of the transmission probability. Interestingly, our estimate is lower than what others have concluded from the same 
data. ${ }^{9}$ Seventy per cent of female and $68 \%$ of male partners were infected with chlamydia if their sexual partner was also chlamydia-positive. These values have often, but incorrectly, been interpreted as the per partnership transmission probabilities. ${ }^{16,17}$ However, the direction of transmission cannot be reliably determined from these raw percentages. ${ }^{6}$ This discrepancy illustrates the importance of taking into account the natural history of chlamydia infection and the dynamics of sexual partnership formation in estimating transmissibility from data of chlamydia-positivity in couples.

The estimates of the per sex act transmission probability for chlamydia are consistent with those obtained or used in other modeling studies ${ }^{18-20}$. While the estimates are lower than for Neisseria gonorrhoeae ${ }^{4,21}$ they are considerably higher than the per heterosexual sex act transmission probability for HIV. ${ }^{22,23}$ Our estimates of the per sex act transmission probability are limited by the lack of information on the number of unprotected episodes of heterosexual intercourse in the couples. While one episode of intercourse every 5 days on average is in good agreement with population-based data of sexual activity, ${ }^{24,25}$ the proportion of unprotected episodes is unknown. An additional limitation is that we could not investigate differences between women and men. Different female-to-male and male-to-female transmission probabilities and a higher proportion of symptomatic cases in men could result in sex-specific differences in the transmission and prevalence of chlamydia. As already noted, however, the data from Quinn et al. showed a strikingly similar chlamydia positivity pattern between women and men. ${ }^{9}$ Ultimately, our estimates are derived from a single study population. While this ensures consistency of our results, the per partnership transmission probability might differ in the general population where partnership durations might be longer than in the study population.

The framework described here does not take into account natural variability in chlamydia transmission. For example, spontaneous resolution of chlamydia could confer a certain degree of immunity ${ }^{26}$ resulting in heterogeneity of susceptibility and transmissibility in a population. Tu et al. present a promising Bayesian framework using detailed longitudinal individual level data about chlamydia infection status and the type and frequency of sexual intercourse in women. ${ }^{20}$ If such longitudinal data sets were matched to the women's male partners' infection status and sexual behavior, more detailed investigations of sex differences in chlamydia transmissibility and its heterogeneity could be performed. In summary, we report the expected ranges of the average per partnership and per sex act chlamydia transmission probability. Fur- 
ther efforts will be needed to investigate the between-individual heterogeneity in susceptibility and transmissibility of chlamydia.

\section{References}

1. Rosen NO, Knäuper B, Mozessohn L, et al. Factors affecting knowledge of sexually transmitted infection transmissibility in healthcare providers: results from a national survey. Sex Transm Dis 2005;32: 619-24.

2. Althaus CL, Turner KME, Schmid BV, et al. Transmission of Chlamydia trachomatis through sexual partnerships: a comparison between three individual-based models and empirical data. J R Soc Interface 2012;9: 136-146.

3. Datta SD, Sternberg M, Johnson RE, et al. Gonorrhea and chlamydia in the United States among persons 14 to 39 years of age, 1999 to 2002. Ann Intern Med 2007;147: 89-96.

4. Lycke E, Löwhagen GB, Hallhagen G, et al. The risk of transmission of genital Chlamydia trachomatis infection is less than that of genital Neisseria gonorrhoeae infection. Sex Transm Dis 1980;7: 6-10.

5. Katz BP. Estimating transmission probabilities for chlamydial infection. Stat Med 1992; 11: $565-577$.

6. Garnett GP and Bowden FJ. Epidemiology and control and curable sexually transmitted diseases: opportunities and problems. Sex Transm Dis 2000;27: 588-99.

7. Althaus CL, Heijne JC, Roellin A, et al. Transmission dynamics of Chlamydia trachomatis affect the impact of screening programmes. Epidemics 2010;2: 123 - 131.

8. Heijne JCM, Althaus CL, Herzog SA, et al. The role of reinfection and partner notification in the efficacy of Chlamydia screening programs. J Infect Dis 2011;203: 372-7.

9. Quinn TC, Gaydos C, Shepherd M, et al. Epidemiologic and microbiologic correlates of Chlamydia trachomatis infection in sexual partnerships. JAMA 1996;276: 1737-42.

10. Dietz K and Hadeler KP. Epidemiological models for sexually transmitted diseases. J Math Biol 1988;26: 1-25. 
11. Kretzschmar M, Jager JC, Reinking DP, et al. The basic reproduction ratio R0 for a sexually transmitted disease in a pair formation model with two types of pairs. Math Biosci 1994;124: 181-205.

12. Chen MI, Ghani AC, and Edmunds J. Mind the gap: the role of time between sex with two consecutive partners on the transmission dynamics of gonorrhea. Sex Transm Dis 2008;35: $435-444$.

13. Bolker BM. Ecological Models and Data in R. Princeton, NJ: Princeton University Press, 2008. ISBN: 0691125228.

14. R Development Core Team. R: A Language and Environment for Statistical Computing. R Foundation for Statistical Computing, Vienna, Austria, 2009.

15. Akaike H. A new look at the statistical model identification. Automatic Control, IEEE Transactions on 1974;19: $716-723$.

16. Kretzschmar M, Welte $\mathrm{R}$, van den Hoek A, et al. Comparative model-based analysis of screening programs for Chlamydia trachomatis infections. Am J Epidemiol 2001;153: $90-101$.

17. Welte R, Postma M, Leidl R, et al. Costs and effects of chlamydial screening: dynamic versus static modeling. Sex Transm Dis 2005;32: 474-483.

18. Regan DG, Wilson DP, and Hocking JS. Coverage is the key for effective screening of Chlamydia trachomatis in Australia. J Infect Dis 2008;198: 349-358.

19. Johnson LF, Alkema L, and Dorrington RE. A Bayesian approach to uncertainty analysis of sexually transmitted infection models. Sex Transm Infect 2010;86: 169-74.

20. Tu W, Ghosh P, and Katz BP. A Stochastic Model for Assessing Chlamydia trachomatis Transmission Risk Using Longitudinal Observational Data. J R Stat Soc Ser A Stat Soc 2011;174: 975-989.

21. Hooper RR, Reynolds GH, Jones OG, et al. Cohort study of venereal disease. I: the risk of gonorrhea transmission from infected women to men. Am J Epidemiol 1978;108: 136-44. 
22. Quinn TC, Wawer MJ, Sewankambo N, et al. Viral load and heterosexual transmission of human immunodeficiency virus type 1. Rakai Project Study Group. N Engl J Med 2000; 342: $921-9$.

23. Attia S, Egger M, Müller M, et al. Sexual transmission of HIV according to viral load and antiretroviral therapy: systematic review and meta-analysis. AIDS 2009;23: 1397-404.

24. Blower SM and Boe C. Sex acts, sex partners, and sex budgets: implications for risk factor analysis and estimation of HIV transmission probabilities. J Acquir Immune Defic Syndr 1993;6: $1347-52$.

25. Johnson AM, Mercer CH, Erens B, et al. Sexual behaviour in Britain: partnerships, practices, and HIV risk behaviours. Lancet 2001;358: 1835-1842.

26. Batteiger BE, Xu F, Johnson RE, et al. Protective immunity to Chlamydia trachomatis genital infection: evidence from human studies. J Infect Dis 2010;201 Suppl 2: S178-89. 


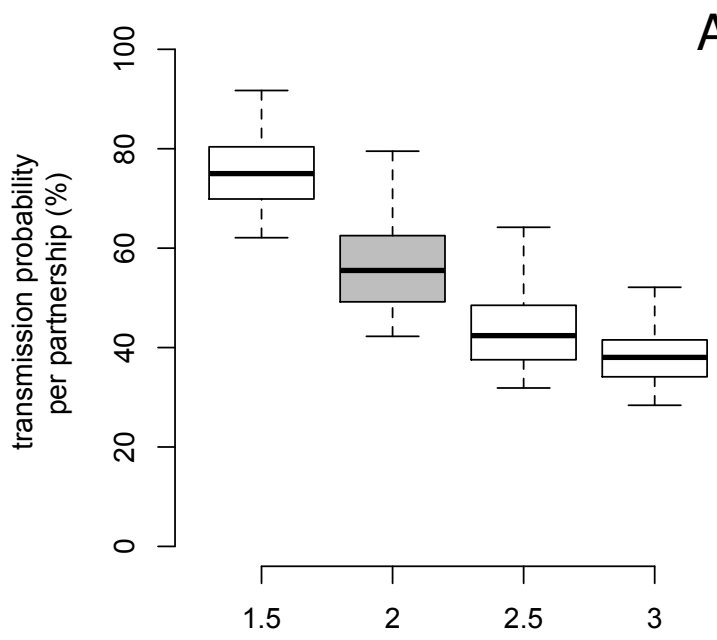

heterosexual partners in last 6 months

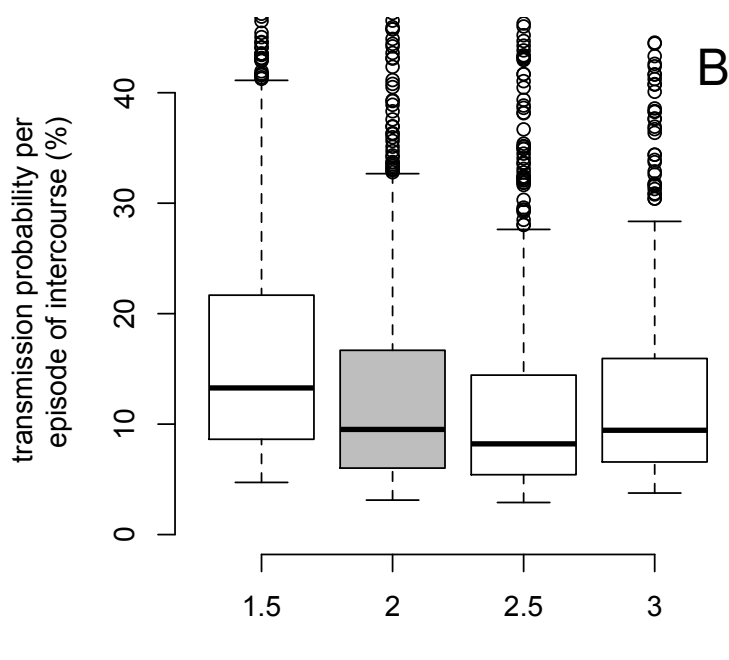

heterosexual partners in last 6 months

Figure 1: Estimated transmission probabilities for different values of the number of partners in the last 6 months. (A) Per partnership transmission probability of chlamydia. (B) Per sex act transmission probability of chlamydia. Each boxplot represents estimates from 1000 different parameter combinations. The baseline scenario, where it is assumed that individuals in a partnership at steady-state have on average 2 heterosexual partners during the previous 6 months, is in gray. It is assumed that individuals have one episode of heterosexual intercourse every 5 days. 